\title{
Haematopoietic stem cells do not asymmetrically segregate chromosomes or retain BrdU
}

\author{
Mark J. Kiel ${ }^{1}$, Shenghui He ${ }^{1}$, Rina Ashkenazi ${ }^{2}$, Sara N. Gentry ${ }^{2}$, Monica Teta ${ }^{3}$, Jake A. Kushner ${ }^{3}$, Trachette L. Jackson $^{2}$ \\ $\&$ Sean J. Morrison ${ }^{1}$
}

Stem cells are proposed to segregate chromosomes asymmetrically during self-renewing divisions so that older ('immortal') DNA strands are retained in daughter stem cells whereas newly synthesized strands segregate to differentiating cells ${ }^{1-6}$. Stem cells are also proposed to retain DNA labels, such as 5-bromo-2deoxyuridine (BrdU), either because they segregate chromosomes asymmetrically or because they divide slowly ${ }^{5,7-9}$. However, the purity of stem cells among BrdU-label-retaining cells has not been documented in any tissue, and the 'immortal strand hypothesis' has not been tested in a system with definitive stem cell markers. Here we tested these hypotheses in haematopoietic stem cells (HSCs), which can be highly purified using well characterized markers. We administered BrdU to newborn mice, mice treated with cyclophosphamide and granulocyte colony-stimulating factor, and normal adult mice for 4 to 10 days, followed by 70 days without BrdU. In each case, less than $6 \%$ of HSCs retained BrdU and less than $0.5 \%$ of all BrdU-retaining haematopoietic cells were HSCs, revealing that BrdU has poor specificity and poor sensitivity as an HSC marker. Sequential administration of 5-chloro-2deoxyuridine and 5-iodo-2-deoxyuridine indicated that all HSCs segregate their chromosomes randomly. Division of individual HSCs in culture revealed no asymmetric segregation of the label. Thus, HSCs cannot be identified on the basis of BrdU-label retention and do not retain older DNA strands during division, indicating that these are not general properties of stem cells.

The immortal strand hypothesis was proposed as a mechanism by which stem cells could avoid accumulating mutations that arise during DNA replication ${ }^{2}$. Whereas most cells segregate their chromosomes randomly ${ }^{1,10}$, it was argued that adult stem cells in steady-state tissues might retain older DNA strands during asymmetric selfrenewing divisions, segregating newly synthesized strands to daughter cells fated to differentiate (Fig. 1a). Evidence has supported this model in some epithelial stem cells ${ }^{1}$, neural stem cells ${ }^{3}$, mammary epithelial progenitors ${ }^{4}$ and muscle satellite cells $s^{5,6}$. A related idea is that adult stem cells in steady-state tissues might consistently retain DNA labels. This could be because chromosomes segregate randomly but stem cells divide more infrequently than other cells (Fig. 1b), or alternatively because the older DNA strand is labelled and segregated asymmetrically (Fig. 1c). Tritiated thymidine ${ }^{8}$ or histone $e^{7}$ labelretaining cells from the hair follicle are enriched for epithelial stem cells, although the purity remains uncertain. Label-retaining cells have also been identified in the haematopoietic system ${ }^{9,11}$, in mammary epithelium ${ }^{12}$, in intestinal epithelium ${ }^{1,13}$ and in the heart ${ }^{14}$, but the purity of stem cells among these label-retaining cells has not been tested. As a result, it remains unclear whether label retention can consistently identify stem cells with specificity or sensitivity.
Under steady-state conditions in adult bone marrow, all HSCs divide regularly but infrequently ${ }^{15}$ to sustain haematopoiesis and to maintain nearly constant numbers of HSCs. As a result of this observation, as well as the finding that HSC divisions yield asymmetric outcomes in culture ${ }^{16}$, it has been proposed that adult HSCs divide asymmetrically ${ }^{16}$, although the rarity of HSCs in vivo and their

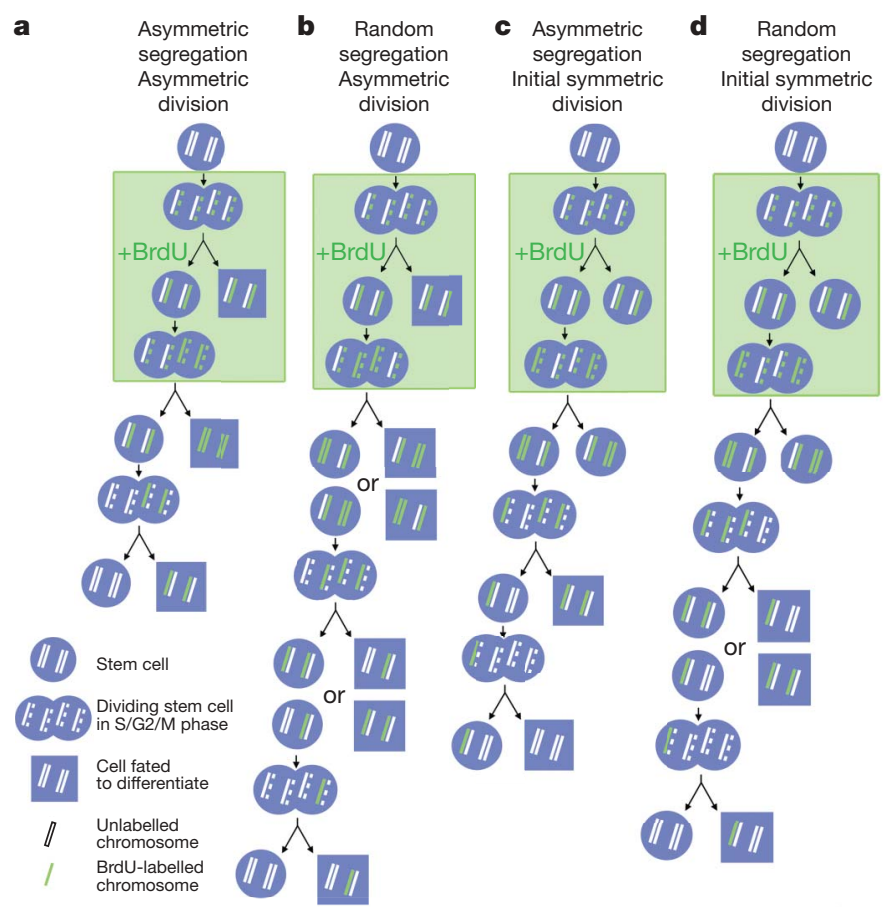

Figure 1 | Contrasting predictions regarding stem cell labelling on the basis of the immortal strand model versus random chromosome segregation. a, According to the immortal strand model ${ }^{2}$, stem cells divide asymmetrically under steady-state conditions and BrdU is incorporated into newly synthesized DNA strands that are asymmetrically segregated into differentiating daughter cells with each round of division, such that stem cells retain only the unlabelled older DNA strands. b, In contrast, if chromosomes segregate randomly, then BrdU-labelled chromosomes will be stochastically lost over multiple rounds of divisions. c, In the immortal strand model, if stem cells divide symmetrically then BrdU can be incorporated into DNA strands that become the 'older' strands once stem cells resume asymmetric division. Under these circumstances, the $\mathrm{BrdU}^{+}$ older strands would be retained indefinitely in stem cells. $d$, In contrast, if chromosome segregation is random then $\mathrm{BrdU}^{+}$chromosomes are stochastically lost over time after BrdU is discontinued. 
relative quiescence has made it impossible to confirm this directly. Nonetheless, if BrdU-label retention and/or asymmetric chromosome segregation are general properties of adult stem cells, then either or both of these characteristics should be evident in HSCs, depending on experimental conditions.

To test the rate at which HSCs enter the cell cycle we administered BrdU to mice for 1, 4 or 10 days, and then sorted HSCs onto microscope slides and stained with an anti-BrdU antibody. HSCs were sorted as $\mathrm{CD} 150^{+} \mathrm{CD} 48^{-} \mathrm{CD} 41^{-}$lineage ${ }^{-} \mathrm{Sca}-1^{+}{ }^{\mathrm{c}-\mathrm{kit}^{+}}$cells (Fig. 2a). This population contains all of the detectable HSC activity in bone marrow, and $47 \%$ of single cells from this population give long-term multilineage reconstitution in irradiated mice ${ }^{17}$. After $1-10$ days of BrdU treatment, 51-94\% of whole bone marrow cells and $6.5-45.9 \%$ of CD $150^{+} \mathrm{CD} 48^{-}$ CD41 ${ }^{-}$lineage ${ }^{-} \mathrm{Sca}-1^{+} \mathrm{c}^{-\mathrm{kit}^{+}}{ }^{+} \mathrm{HSC}$ became BrdU ${ }^{+}$(Fig. 2c, d). We calculated the rate at which HSCs entered the cell cycle ${ }^{15}$ to be $6.0 \%$ per day (Fig. 2e). Consistent with this, only $3.2 \%$ of $\mathrm{CD} 150^{+} \mathrm{CD} 48^{-} \mathrm{CD} 41^{-}$ lineage ${ }^{-} \mathrm{Sca}-1^{+} \mathrm{c}^{-\mathrm{kit}^{+}}$cells were in S/G2/M phase of the cell cycle at any one time (Supplementary Fig. 3). These results are similar to a previous study that identified HSCs using different markers ${ }^{15}$.

The linearity of BrdU incorporation over time (Fig. 2e) suggests that most HSCs divide at a similar rate. If a minority of HSCs divided more rapidly, consistently more than $6.0 \%$ of HSCs should have incorporated BrdU after one day; however, we did not observe this (Fig. 2d). If a minority of HSCs were more deeply quiescent than most other HSCs, these HSCs should remain BrdU-negative, even after long periods of BrdU treatment. This also has not been observed, because more than $99 \%$ of HSCs are labelled after 6 months of BrdU treatment ${ }^{15}$. Therefore, there is no evidence for more rapidly dividing or

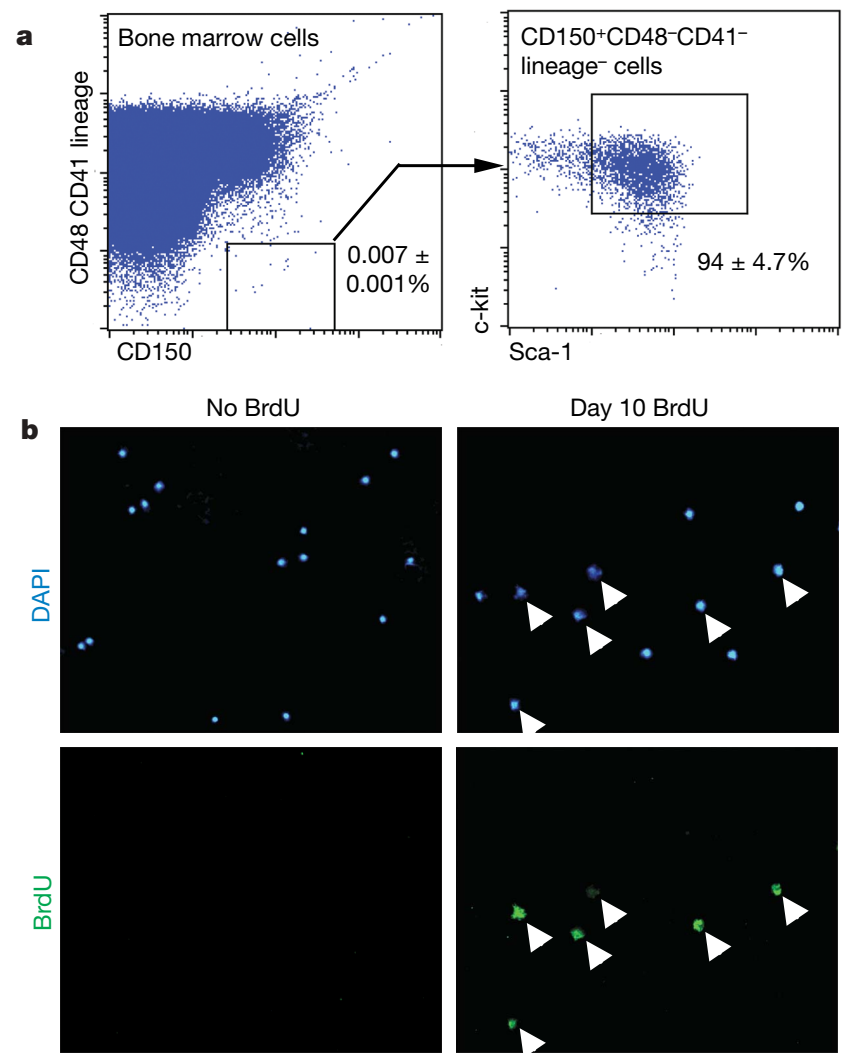

Figure 2 | Six per cent of HSCs stochastically enter the cell cycle each day. a, HSCs can be isolated by flow cytometry as

$\mathrm{CD} 150^{+} \mathrm{CD} 48^{-} \mathrm{CD} 41^{-}$lineage ${ }^{-} \mathrm{Sca}-1^{+} \mathrm{c}$-kit ${ }^{+}$cells; these represent only $0.0066 \pm 0.0003 \%(0.007 \% \times 94 \%)$ of bone marrow cells but contain all detectable HSC activity and are very highly enriched for HSCs (nearly $50 \%$ of single cells give long-term multilineage reconstitution in irradiated mice $^{17}$ ). b, BrdU incorporation into HSCs (arrowheads) is evaluated by immunofluorescence after sorting HSCs onto microscope slides (DAPI is a more slowly dividing subsets of long-term self-renewing HSCs under steady-state conditions, although we cannot exclude the possibility that a minority of HSCs divide more slowly than $6.0 \%$ per day.

To evaluate retention of the BrdU label, we administered BrdU for 10 days, followed by 70 days without BrdU (a 70-day 'chase'), like previous studies in the haematopoietic system ${ }^{9,11}$. Given that $6.0 \%$ of HSCs entered the cell cycle each day and $46 \%$ of HSCs were labelled after 10 days of BrdU treatment (Fig. 2d), we modelled the fraction of HSCs that would be expected to retain BrdU over time (Fig. 3a; see Methods for explanation).

Under these experimental conditions, if HSCs follow the immortal strand model they should lose their BrdU label one division after $\mathrm{BrdU}$ is discontinued because the labelled chromosomes would be segregated to differentiating daughter cells (Fig. 1a); therefore, only $0.6 \%$ of HSCs would be expected to retain BrdU after the 70-day chase (Fig. 3a). If HSCs segregate chromosomes randomly, then BrdU would be lost stochastically over time and the fraction of $\mathrm{BrdU}^{+}$HSCs after a 70-day chase would depend on the threshold of $\mathrm{BrdU}$ required for detection. If the threshold is equivalent to $0.5 n$ labelled chromosomes (one-quarter of the genome), this level of BrdU dilution could be achieved in cells that had divided 1 or 2 times after BrdU was discontinued (depending on whether they had divided once or twice in the presence of BrdU), and only $1.4 \%$ of HSCs would be expected to retain BrdU after the 70-day chase (Fig. 3a). In contrast, if the threshold of detection is equivalent to $0.0625 n$ labelled chromosomes, this could be achieved on average in cells that had divided 4 or 5 times after BrdU was discontinued, and $19.8 \%$ of HSCs would be expected to retain BrdU after the 70-day

c BrdU+ bone marrow cells (\%)

\begin{tabular}{ccccc}
\hline Experiment & Day 0 & Day 1 & Day 4 & Day 10 \\
\hline 1 & 0.0 & 49.6 & 78.3 & 92.0 \\
2 & 0.0 & 49.3 & 91.0 & 96.5 \\
3 & 0.0 & 53.6 & & 93.3 \\
Mean \pm s.d. & $0.0 \pm 0.0$ & $51 \pm 2.4$ & 85 & $94 \pm 2.3$ \\
\hline
\end{tabular}

d BrdU+ HSCs (\%)

\begin{tabular}{ccccc}
\hline Experiment & Day 0 & Day 1 & Day 4 & Day 10 \\
\hline 1 & 0.0 & 4.5 & 25.0 & 44.6 \\
2 & 0.0 & 5.6 & 23.6 & 43.2 \\
3 & 0.0 & 5.0 & & 50.0 \\
4 & 0.0 & 10.9 & & \\
Mean \pm s.d. & $0.0 \pm 0.0$ & $6.5 \pm 3.0$ & 24 & $46 \pm 3.6$
\end{tabular}

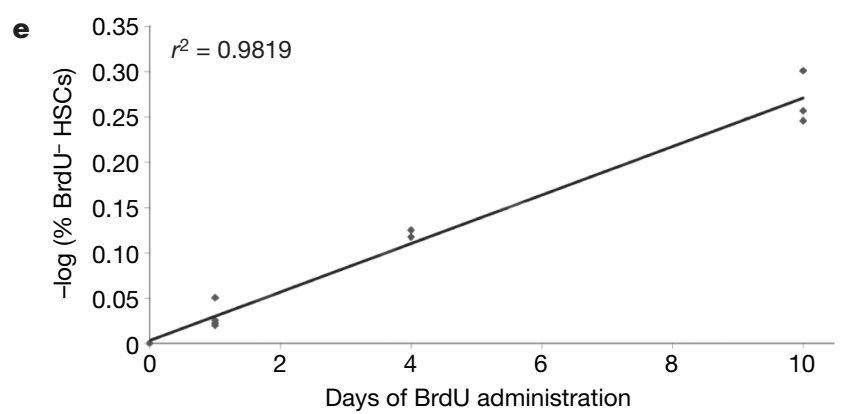

nuclear stain). c, d, The percentage of $\mathrm{BrdU}^{+}$bone marrow cells (c) and $\mathrm{CD} 50^{+} \mathrm{CD}^{-} 8^{-} \mathrm{CD} 1^{-}$lineage ${ }^{-} \mathrm{Sca}-1^{+} \mathrm{c}^{-\mathrm{kit}^{+}}$HSCs (d) after various periods of BrdU administration (3-4 independent experiments with 3-4 mice per experiment and 200-400 bone marrow cells or 100-400 HSCs counted, respectively, per mouse). Standard deviations are shown for means that are based on at least three independent experiments. e, The percentage of HSCs that enter the cell cycle each day $(6 \%)$ can be derived by plotting the negative logarithm of the percentage of HSCs that were BrdU ${ }^{-}$over time ${ }^{15}$. 
chase (Fig. 3a). Thus, these calculations predict that few $(<20 \%)$ HSCs should retain BrdU after a 70-day chase, irrespective of how chromosomes are segregated.

To test this we administered BrdU for 10 days to adult mice and then stained whole bone marrow cells and $\mathrm{CD} 150^{+} \mathrm{CD} 48^{-} \mathrm{CD} 41^{-}$ lineage ${ }^{-} \mathrm{Sca}-1^{+} \mathrm{c}^{-k i t^{+}}$HSCs after 40, 70 and 120 days of chase. After 70 days of chase, $0.4 \pm 0.1 \%$ (mean \pm s.d.) of bone marrow cells and $4.6 \pm 0.9 \%$ of HSCs were $\mathrm{BrdU}^{+}$(Fig. 3c). This demonstrates that, as predicted (Fig. 3a), few HSCs retain BrdU. Moreover, only $2.0 \pm 1.0 \%$ of HSCs were $\mathrm{BrdU}^{+}$after 120 days of chase, demonstrating that the frequency of BrdU-retaining HSCs continues to decline over time rather than there being a deeply quiescent subset of HSCs that retains BrdU indefinitely. Although BrdU-label-retaining cells were enriched in HSCs by tenfold compared to whole bone marrow cells, the rarity of HSCs means that only $0.08 \%$ of $\mathrm{BrdU}^{+}$bone marrow cells were HSCs $(0.0066 \% \times 4.6 \% / 0.4 \%)$. BrdU-label retention is therefore a very insensitive and nonspecific marker of HSCs because most HSCs did not retain detectable BrdU, and only rare BrdU-label-retaining cells were HSCs. Very similar results were obtained when we used flow cytometry to detect BrdU incorporation (Supplementary Fig. 1) or when HSCs were isolated using different surface markers (c-kit ${ }^{+}$Flk-2- ${ }^{-}$ineage ${ }^{-} \mathrm{Sca}-1^{+}$cells; Supplementary Fig. 2). These data are most consistent with random chromosome segregation by HSCs and the failure to detect BrdU after approximately three divisions in the absence of BrdU (Fig. 3a).

According to the immortal strand model, stem cells can incorporate BrdU into DNA strands that become the 'older' strands during symmetric cell divisions, and these labelled DNA strands would be retained indefinitely by stem cells that resume asymmetric divisions
(Fig. 1c). To test this, we administered BrdU to newborn mice for 10 days or to cyclophosphamide and granulocyte colony-stimulating factor (cyclophosphamide/G-CSF)-treated mice for 4 days. The absolute number of HSCs expands markedly in both newborn ${ }^{18}$ and cyclophosphamide/G-CSF-mobilized mice ${ }^{19}$ (indicating symmetric divisions), before stabilizing to steady-state levels as mice enter adulthood or after G-CSF is discontinued. After 10 days of BrdU treatment in neonatal mice, $93 \pm 3.7 \%$ of bone marrow cells and $80 \pm 11 \%$ of $\mathrm{CD} 150^{+} \mathrm{CD} 48^{-} \mathrm{CD} 41^{-}$lineage ${ }^{-} \mathrm{Sca}-1^{+}{ }^{+}$-kit ${ }^{+}$HSCs were $\mathrm{BrdU}^{+}$. Seventy days later, $0.1 \%$ of bone marrow cells and $6.4 \pm 2.5 \%$ of $\mathrm{CD} 150^{+} \mathrm{CD} 48^{-} \mathrm{CD} 41^{-}$lineage ${ }^{-} \mathrm{Sca}-1^{+} \mathrm{c}^{-\mathrm{kit}^{+}}$HSCs were $\mathrm{BrdU}^{+}$(Fig. 3d). After 4 days of BrdU treatment in cyclophosphamide/G-CSF mobilized mice, $94 \pm 3 \%$ of $\mathrm{CD} 150^{+} \mathrm{CD} 48^{-} \mathrm{CD} 41^{-}$ lineage $\mathrm{Sca}^{-} \mathrm{1}^{+} \mathrm{c}_{\text {-kit }}{ }^{+}$HSCs were $\mathrm{BrdU}^{+}$. Seventy days later, $0.1 \%$ of bone marrow cells and $2.1 \pm 1.1 \%$ of $\mathrm{CD} 150^{+} \mathrm{CD}^{-} 8^{-} \mathrm{CD} 41^{-}$lineage Sca- ${ }^{+}{ }^{\text {c-kit }}{ }^{+}$HSCs were BrdU ${ }^{+}$(Fig. 3d). Thus, even when BrdU was administered to symmetrically dividing HSCs, only $2-6 \%$ of HSCs retained the label and only $0.2-0.4 \%$ of BrdU-retaining bone marrow cells were HSCs. We were unable to identify any context in which BrdU-label retention identified HSCs with sensitivity or specificity, and none of these results was consistent with the immortal strand hypothesis.

To address the possibility that the HSCs in the above experiments might have continued to divide symmetrically after BrdU was discontinued, we also administered BrdU to mice from 20 to 29 days postnatally. HSCs are thought to transition from rapidly dividing cells that have a fetal phenotype to relatively quiescent cells that have an adult phenotype between 21 and 28 days postnatally ${ }^{18}$. We obtained similar results, with only $6.5 \pm 1.1 \%$ of HSCs retaining

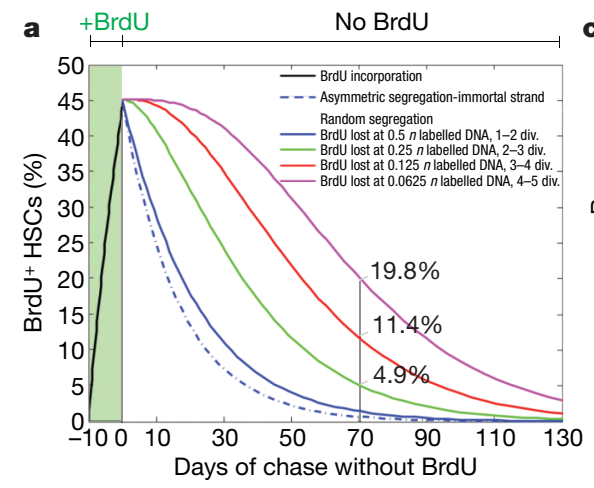

\begin{tabular}{|c|c|c|c|c|}
\hline \multirow[t]{2}{*}{ c } & \multicolumn{4}{|c|}{ BrdU+ cells from adult mice (\%) } \\
\hline & Experiment & $\begin{array}{c}40 \text { days } \\
\text { chase }\end{array}$ & $\begin{array}{c}70 \text { days } \\
\text { chase }\end{array}$ & $\begin{array}{c}120 \text { days } \\
\text { chase }\end{array}$ \\
\hline \multirow{5}{*}{ 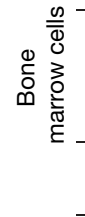 } & 1 & 0.3 & 0.4 & 0.3 \\
\hline & 2 & 0.6 & 0.3 & 0.4 \\
\hline & 3 & & 0.5 & 0.1 \\
\hline & Mean \pm s.d. & 0.4 & $0.4 \pm 0.1$ & $0.3 \pm 0.1$ \\
\hline & Experiment & $\begin{array}{c}40 \text { days } \\
\text { chase }\end{array}$ & $\begin{array}{l}70 \text { days } \\
\text { chase }\end{array}$ & $\begin{array}{c}120 \text { days } \\
\text { chase }\end{array}$ \\
\hline \multirow{6}{*}{$\begin{array}{l}0 \\
0 \\
\text { I }\end{array}$} & 1 & 7.4 & 4.0 & 1.5 \\
\hline & 2 & 14.5 & 5.7 & 1.4 \\
\hline & 3 & 8.3 & 3.4 & 3.1 \\
\hline & 4 & 6.9 & 5.0 & \\
\hline & 5 & & 4.8 & \\
\hline & Mean \pm s.d. & $9.3 \pm 3.5$ & $4.6 \pm 0.9$ & $2.0 \pm 1.0$ \\
\hline
\end{tabular}

b

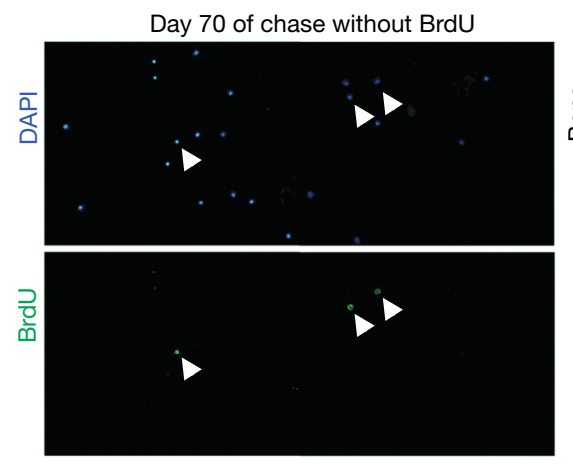

d $\quad \mathrm{BrdU}^{+}$cells from neonatal or mobilized mice (\%)

70 days chase 120 days chase 70 days chase

\begin{tabular}{|c|c|c|c|c|}
\hline \multirow{5}{*}{ 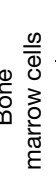 } & Experiment & $\begin{array}{c}70 \text { days chase } \\
\text { neonate }\end{array}$ & $\begin{array}{c}120 \text { days chase } \\
\text { neonate }\end{array}$ & $\begin{array}{c}70 \text { days chase } \\
\text { mobilized }\end{array}$ \\
\hline & 1 & 0.0 & 0.1 & 0.0 \\
\hline & 2 & 0.2 & 0.1 & 0.0 \\
\hline & 3 & 0.2 & 0.0 & 0.2 \\
\hline & Mean \pm s.d. & $0.1 \pm 0.1$ & $0.1 \pm 0.1$ & $0.1 \pm 0.1$ \\
\hline \multirow{7}{*}{$\begin{array}{l}\mathscr{O} \\
\text { O } \\
\text { I }\end{array}$} & Experiment & $\begin{array}{l}70 \text { days chase } \\
\text { neonate }\end{array}$ & $\begin{array}{c}120 \text { days chase } \\
\text { neonate }\end{array}$ & $\begin{array}{c}70 \text { days chase } \\
\text { mobilized }\end{array}$ \\
\hline & 1 & 6.1 & 2.5 & 2.8 \\
\hline & 2 & 5.6 & 0.8 & 2.6 \\
\hline & 3 & 4.3 & 2.1 & 0.9 \\
\hline & 4 & 10.8 & & \\
\hline & 5 & 5.3 & & \\
\hline & Mean \pm s.d. & $6.4 \pm 2.5$ & $1.8 \pm 0.9$ & $2.1 \pm 1.1$ \\
\hline
\end{tabular}

Figure 3 | Few HSCs retain BrdU, and most BrdU-retaining bone marrow cells are not HSCs. a, A model is shown that predicts the fraction of HSCs that retain BrdU over time after administering BrdU for 10 days, depending on whether chromosomes segregate asymmetrically or randomly and on the threshold of BrdU that can be detected by immunofluorescence $(0.5 n, 0.25 n$, $0.125 n$ or $0.0625 n$ labelled DNA). b, CD $150^{+} \mathrm{CD} 48^{-} \mathrm{CD} 41^{-}$lineage ${ }^{-} \mathrm{Sca}-1^{+}$

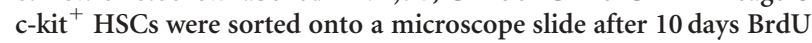
administration and 70 days chase (without BrdU). Arrowheads identify $\mathrm{BrdU}^{+}$cells. c, Shown is the frequency of BrdU ${ }^{+}$bone marrow cells and
HSCs after 10 days of BrdU administration and 40, 70 or 120 days of chase. Standard deviations are shown for means that are based on at least three independent experiments. $\mathbf{d}$, Shown is the frequency of $\mathrm{BrdU}^{+}$bone marrow cells and HSCs after 10 days BrdU administration to neonatal mice followed by 70 or 120 days of chase, or after 4 days BrdU administration to cyclophosphamide/G-CSF-mobilized mice followed by 70 days of chase. All data are based on 3-5 independent experiments with 3 mice per experiment and 400-700 bone marrow cells or 300-400 HSCs counted per mouse. 
BrdU after a 70-day chase. There was, therefore, no period during neonatal development when BrdU could be administered in a way that resulted in retention of BrdU within significant numbers of HSCs.

To test the immortal strand model directly, we treated mice with 5-chloro-2-deoxyuridine (CldU) for 10 days and then with 5-iodo-2deoxyuridine (IdU) for 10 days. If HSCs segregate older and younger DNA strands asymmetrically, then HSCs should rarely incorporate both CldU and IdU under steady-state conditions because newly
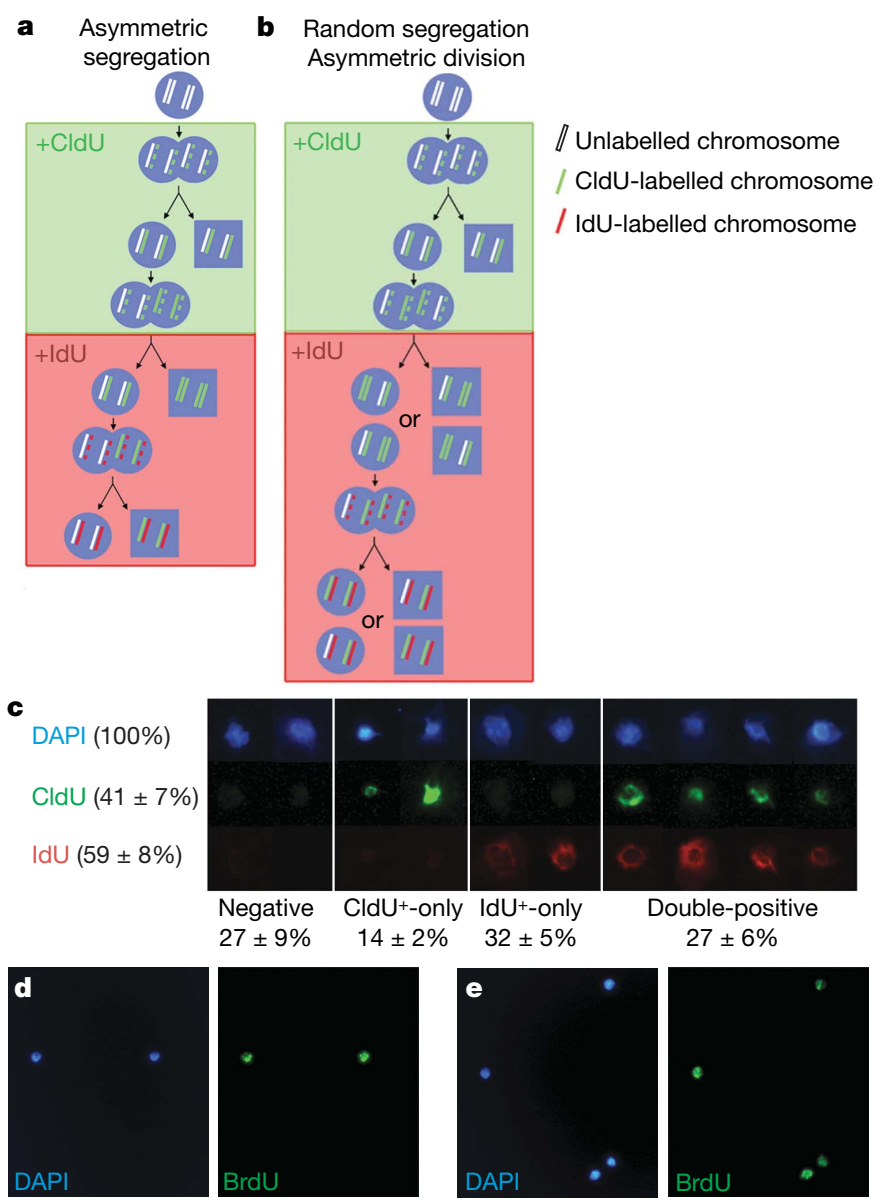

f Single cell culture of BrdU-labeled HSCs

\begin{tabular}{cccc}
\hline $\begin{array}{c}\text { Number of cells } \\
\text { harvested from } \\
\begin{array}{c}\text { individual colony } \\
(>1 \text { cell })\end{array}\end{array}$ & $\begin{array}{c}\text { Colonies } \\
\text { with only } \\
\text { BrdU- cells }\end{array}$ & $\begin{array}{c}\text { Colonies } \\
\text { with only } \\
\text { BrdU+ cells }\end{array}$ & $\begin{array}{c}\text { Colonies with } \\
\text { BrdU+ }^{+} \text {and } \\
\text { BrdU- cells }^{-}\end{array}$ \\
\hline 2 cells & 123 & 86 & 0 \\
3 cells & 38 & 50 & 0 \\
4 cells & 23 & 26 & 0 \\
Total & 184 & 162 & 0 \\
\hline
\end{tabular}

Figure 4 | HSCs segregate chromosomes randomly in vivo and in vitro. a, By the immortal strand model, stem cells sequentially exposed to CldU (for 10 days) and then IdU (for 10 days) would not incorporate both labels, with the exception of rare cells in the S/G2/M phase of their first division after switching from CldU into IdU (expected frequency $<3 \%$ ). $\mathbf{b}$, In contrast, if chromosome segregation is random then $\mathrm{CldU}^{+}$stem cells would have the same probability of incorporating IdU as unlabelled cells (expected frequency of $\mathrm{CldU}^{+} \mathrm{IdU}^{+} \mathrm{HSCs}$ is $\sim 25 \%$ ). c, CldU was administered to mice for 10 days followed by IdU for $10-11$ days, and $\mathrm{CD} 150^{+} \mathrm{CD} 48^{-} \mathrm{CD} 41^{-}$lineage $\mathrm{Sca}-\mathrm{I}^{+}$

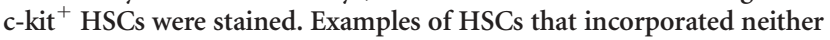
label, only CldU, only IdU or both labels are shown (mean $\pm \mathrm{sd}$; data are based on two independent experiments with 2 or 3 mice per experiment and 100-250 HSCs per mouse). HSCs from BrdU-treated mice divided once (d) or twice (e) in culture to form daughter cells. $f$, The progeny of these HSCs were either all $\mathrm{BrdU}^{+}$or all $\mathrm{BrdU}^{-}$. We detected no clones in which label was asymmetrically segregated to a subset of daughter cells. synthesized (labelled) DNA strands should be segregated to differentiating daughter cells after each division (Fig. 4a). In contrast, if HSCs segregate older and younger DNA strands randomly then CldU-labelled HSCs should have the same chance of incorporating IdU as unlabelled cells, and approximately $25 \%(50 \% \times 50 \%)$ of HSCs should be double-labelled (Fig. 4b).

After 10 days of CldU followed by 10-11 days of IdU, we observed that $14 \%$ of HSCs incorporated only CldU, 32\% incorporated only IdU and 27\% incorporated both CldU and IdU (Fig. 4c and Supplementary Fig. 4). The frequency of $\mathrm{CldU}^{+} \mathrm{IdU}^{+}$cells $(27 \%)$ was therefore similar to the product of the frequencies of total $\mathrm{CldU}^{+}$ cells and total $\mathrm{IdU}^{+}$cells $(41 \% \times 59 \%=24 \%)$, indicating that $\mathrm{CldU}^{+}$cells had a similar probability of incorporating IdU as the other cells. We repeated this experiment by administering mice with CldU for 60 days followed by IdU for 15 days, and found the frequency of $\mathrm{CldU}^{+} \mathrm{IdU}^{+}$cells $(63 \%)$ was again similar to the product of the frequencies of total $\mathrm{CldU}^{+}$cells and total $\mathrm{IdU}^{+}$cells $(73 \% \times 84 \%=61 \%)$. These results were not significantly affected by a slow clearance of CldU from mice, because CldU was cleared in less than 1 day after being discontinued from the drinking water (Supplementary Fig. 5). These observations directly contradict a key prediction made by the immortal strand hypothesis, but are as would be expected by random chromosome segregation.

The foregoing experiments left the formal possibility that if HSCs divide by a combination of symmetric and asymmetric divisions in vivo we might underestimate the frequency of HSCs that retain older DNA strands. To address this we examined the division of individual HSCs in culture that were isolated from mice treated for 10 days with BrdU. Single CD $150^{+} \mathrm{CD} 48^{-} \mathrm{CD} 41^{-}$lineage ${ }^{-} \mathrm{Sca}-{ }^{+}{ }^{+}$- $\mathrm{kit}^{+}$ HSCs were sorted into cultures under conditions in which half of HSC divisions give asymmetric outcomes (daughter cells with different developmental potentials $)^{16}$. After 2-3 days of culture we observed a total of 346 colonies in which HSCs had divided once (2 daughter cells) or twice ( 3 or 4 daughter cells). Either all daughter cells were $\mathrm{BrdU}^{+}$(162 colonies, 46\%) or all daughter cells were $\mathrm{BrdU}^{-}(184$ colonies; Fig. 4f), as would be expected by random chromosome segregation given that $46 \%$ of HSCs incorporate BrdU in vivo after 10 days (Fig. 2 d). We observed no colonies containing a mixture of $\mathrm{BrdU}^{+}$and $\mathrm{BrdU}^{-}$cells after one or two rounds of division. Thus, these in vitro experiments on individual HSCs failed to detect any asymmetric segregation of labelled chromosomes.

Our results were not confounded by the effects of BrdU, CldU or IdU on HSC proliferation, survival or DNA repair. The cell cycle status (Supplementary Fig. 3) and frequency (data not shown) of HSCs was not affected by these treatments. BrdU, CldU and IdU incorporation from DNA repair was not detectable (Supplementary Fig. 3).

Our results indicate that BrdU-label retention is neither a sensitive nor a specific marker of HSCs. Nonetheless BrdU-label retention could be a better marker of stem cells in other tissues. Moreover, histone-green fluorescent protein retention ${ }^{7}$ may do a better job of marking stem cells, including HSCs, because it can be selectively expressed in subsets of progenitors and may be retained with different kinetics than BrdU. Our data demonstrate the need to test the sensitivity and specificity of BrdU and other label-retention markers before assuming they mark stem cells with fidelity.

Our data also demonstrate that the immortal strand model $^{2}$ does not apply to HSCs and cannot be considered a general model of stem cell division. Our data do not address whether stem cells from other tissues asymmetrically segregate chromosomes or whether HSCs segregate a limited number of chromosomes asymmetrically ${ }^{10}$. Nonetheless, asymmetric chromosome segregation cannot be a mechanism by which HSCs avoid accumulating mutations over time.

\section{METHODS SUMMARY}

BrdU, CldU and IdU administration. All experiments used C57BL/ Ka-CD45.2:Thy-1.1 mice. For experiments in adult mice, BrdU (Sigma) was 
administered when the mice were $8-10$ weeks of age. Mice were given an intraperitoneal injection of $100 \mathrm{mg}$ BrdU per kg body mass in Dulbecco's phosphate buffered saline (DPBS; Gibco) and were maintained on $1 \mathrm{mg} \mathrm{ml}^{-1}$ BrdU in the drinking water for 1-10 days. Amber bottles containing BrdU water were changed every 1-3 days. For retention studies, BrdU water was replaced with regular water and the mice were maintained for 40-120 days before analysis.

BrdU injections into neonatal mice were performed as described ${ }^{20}$. Beginning within 3 days after birth, neonatal mice were injected subcutaneously with $50 \mathrm{mg}$ $\mathrm{BrdU}$ per $\mathrm{kg}$ body weight twice daily for 10 days. Mice were weighed every 2 days and the dose of BrdU was adjusted.

To assess BrdU retention after cytokine mobilization, adult mice were injected with cyclophosphamide ( $200 \mathrm{mg} \mathrm{kg}^{-1}$; Bristol-Myers Squibb) and then on each of the 4 subsequent days they were injected with $250 \mathrm{\mu g} \mathrm{kg}^{-1} \mathrm{day}{ }^{-1}$ of human G-CSF (Amgen) ${ }^{19}$. On the fourth day of G-CSF injection, a single intraperitoneal injection of $100 \mathrm{mg} \mathrm{BrdU}$ per $\mathrm{kg}$ body mass was given and the mice were put on $1 \mathrm{mg} \mathrm{ml}^{-1}$ BrdU water for 4 additional days. The mice were then returned to regular water for 70 days before analysis.

For CldU and IdU experiments, mice were given an intraperitoneal injection of $100 \mathrm{mg}$ CldU per kg body mass in DPBS and were maintained on drinking water containing $1 \mathrm{mg} \mathrm{ml}^{-1} \mathrm{CldU}$ (Sigma) for 10 days. Mice were then given an intraperitoneal injection of $100 \mathrm{mg}$ IdU per kg body mass in DPBS, and were switched to drinking water containing $1 \mathrm{mg} \mathrm{ml}^{-1}$ IdU (Sigma) for 10-11 days before being killed.

For details related to the flow cytometric isolation of HSCs and CldU, IdU and BrdU staining, see Methods.

BrdU segregation in cultured HSCs. Single $\mathrm{CD} 150^{+} \mathrm{CD} 48^{-} \mathrm{CD} 41^{-}$lineage ${ }^{-} \mathrm{Sca}-1^{+}$ ${\mathrm{c}-\mathrm{kit}^{+}}^{+}$HSCs were sorted from BrdU-treated mice into a V-bottom 96-well plate containing Stempro-34 medium (Invitrogen) supplemented with $2 \mathrm{mM}$ L-glutamine, $50 \mu \mathrm{M}$ 2-mercaptoethanol (Sigma), murine IL-3 $\left(10 \mathrm{ng} \mathrm{m}^{-1}\right)$, murine SCF $\left(100 \mathrm{ng} \mathrm{ml}^{-1}\right)$ and murine Tpo $\left(100 \mathrm{ng} \mathrm{ml}^{-1}\right.$; all cytokines were obtained from R\&D Systems) with 10\% charcoal absorbed fetal bovine serum (Cocalico Biologicals Inc.), and were cultured for 2-3 days in low-oxygen chambers $^{21}$. For analysis, plates were centrifuged at $500 \mathrm{~g}$ for $10 \mathrm{~min}$; cells from each colony were then pipetted onto individual wells of Teflon-printed glass slides and were allowed to dry overnight before staining for BrdU and 4,6diamidino-2-phenylindole (DAPI).

Full Methods and any associated references are available in the online version of the paper at www.nature.com/nature.

Received 14 June; accepted 25 July 2007.

Published online 29 August 2007.

1. Potten, C. S., Hume, W. J., Reid, P. \& Cairns, J. The segregation of DNA in epithelial stem cells. Cell 15, 899-906 (1978).

2. Cairns, J. Mutation selection and the natural history of cancer. Nature 255, 197-200 (1975).

3. Karpowicz, P. et al. Support for the immortal strand hypothesis: neural stem cells partition DNA asymmetrically in vitro. J. Cell Biol. 170, 721-732 (2005).

4. Smith, G. H. Label-retaining epithelial cells in mouse mammary gland divide asymmetrically and retain their template DNA strands. Development 132, 681-687 (2005).

5. Shinin, V., Gayraud-Morel, B., Gomes, D. \& Tajbakhsh, S. Asymmetric division and cosegregation of template DNA strands in adult muscle satellite cells. Nature Cell Biol. 8, 677-687 (2006).

6. Conboy, M. J., Karasov, A. O. \& Rando, T. A. High incidence of non-random template strand segregation and asymmetric fate determination in dividing stem cells and their progeny. PLoS Biol. 5, e102 (2007).
7. Tumbar, T. et al. Defining the epithelial stem cell niche in skin. Science 303, 359-363 (2004).

8. Cotsarelis, G., Sun, T. T. \& Lavker, R. M. Label-retaining cells reside in the bulge area of pilosebaceous unit: implications for follicular stem cells, hair cycle, and skin carcinogenesis. Cell 61, 1329-1337 (1990).

9. Zhang, J. et al. Identification of the haematopoietic stem cell niche and control of the niche size. Nature 425, 836-841 (2003).

10. Armakolas, A. \& Klar, A. J. Cell type regulates selective segregation of mouse chromosome 7 DNA strands in mitosis. Science 311, 1146-1149 (2006).

11. Arai, F. et al. Tie2/angiopoietin-1 signaling regulates hematopoietic stem cell quiescence in the bone marrow niche. Cell 118, 149-161 (2004).

12. Welm, B. E. et al. Sca- $1^{+}$cells in the mouse mammary gland represent an enriched progenitor cell population. Dev. Biol. 245, 42-56 (2002).

13. Potten, C. S., Owen, G. \& Booth, D. Intestinal stem cells protect their genome by selective segregation of template DNA strands. J. Cell Sci. 115, 2381-2388 (2002).

14. Urbanek, K. et al. Stem cell niches in the adult mouse heart. Proc. Natl Acad. Sci. USA 103, 9226-9231 (2006).

15. Cheshier, S. H., Morrison, S. J., Liao, X. \& Weissman, I. L. In vivo proliferation and cell cycle kinetics of long-term self-renewing hematopoietic stem cells. Proc. Natl Acad. Sci. USA 96, 3120-3125 (1999).

16. Takano, H., Ema, H., Sudo, K. \& Nakauchi, H. Asymmetric division and lineage commitment at the level of hematopoietic stem cells: inference from differentiation in daughter cell and granddaughter cell pairs. J. Exp. Med. 199, 295-302 (2004)

17. Kiel, M. J., Yilmaz, O. H., Iwashita, T., Terhorst, C. \& Morrison, S. J. SLAM family receptors distinguish hematopoietic stem and progenitor cells and reveal endothelial niches for stem cells. Cell 121, 1109-1121 (2005).

18. Bowie, M. B. et al. Hematopoietic stem cells proliferate until after birth and show a reversible phase-specific engraftment defect. J. Clin. Invest. 116, 2808-2816 (2006).

19. Morrison, S. J., Wright, D. \& Weissman, I. L. Cyclophosphamide/granulocyte colony-stimulating factor induces hematopoietic stem cells to proliferate prior to mobilization. Proc. Natl Acad. Sci. USA 94, 1908-1913 (1997).

20. Taylor, G., Lehrer, M. S., Jensen, P. J., Sun, T. T. \& Lavker, R. M. Involvement of follicular stem cells in forming not only the follicle but also the epidermis. Cell 102, 451-461 (2000).

21. Morrison, S. J. et al. Culture in reduced levels of oxygen promotes clonogenic sympathoadrenal differentiation by isolated neural crest stem cells. J. Neurosci. 20, 7370-7376 (2000).

Supplementary Information is linked to the online version of the paper at www.nature.com/nature.

Acknowledgements This work was supported by the Howard Hughes Medical Institute, the National Institute on Aging (NIH), and the US Army Research Laboratory/Office. Flow cytometry was partially supported by the

UM-Comprehensive Cancer Centre and the UM-Multipurpose Arthritis Centre. Antibody production was partially supported by the Rheumatic Core Disease Centre. M.J.K. was supported by a University of Michigan Cancer Biology Training Grant. The authors thank D. Adams and M. White for flow cytometry and E. Smith (Hybridoma Core Facility) for antibody production.

Author Contributions M.J.K. performed all experiments and interpreted results. S.H. assisted in the design and interpretation of many experiments and helped to perform some experiments. R.A., S.N.G. and T.L.J. generated the mathematical model of BrdU retention over time (Fig. 3a). M.T. and J.A.K. developed the protocol for double-labelling cells with CldU and IdU. S.J.M. participated in the design and interpretation of experiments, and wrote the paper with M.J.K. and S.H.

Author Information Reprints and permissions information is available at www.nature.com/reprints. The authors declare no competing financial interests. Correspondence and requests for materials should be addressed to S.J.M. (seanjm@umich.edu). 


\section{METHODS}

Flow cytometric isolation of HSCs. Bone marrow cells were flushed from the long bones (tibias and femurs) with Hank's buffered salt solution (HBSS) without calcium or magnesium supplemented with $2 \%$ heat-inactivated calf serum (GIBCO). Cells were triturated and filtered through a nylon screen $(45 \mu \mathrm{m}$, Sefar America) to obtain a single-cell suspension. For isolation of Flk2 $2^{-}$lineage ${ }^{-} \mathrm{Sca}-1^{+}$ c-kit $^{+}$HSCs, whole bone marrow cells were incubated with phycoerythrinconjugated monoclonal antibodies against lineage markers including B220 (6B2), CD3 (KT31.1), CD4 (GK1.5), CD8 (53-6.7), Gr-1 (8C5), Mac-1 (M1/70), Flk-2 (A2F10.1), Ter119 and IgM in addition to fluorescein isothiocyanate (FITC)conjugated anti-Sca-1 (Ly6A/E) and biotin-conjugated anti-c-kit (2B8) antibodies, followed by streptavidin allophycocyanin (APC)-Cy7. For isolation of CD $150^{+}$ CD $48^{-} \mathrm{CD} 41^{-}$lineage ${ }^{-} \mathrm{Sca}-1^{+} \mathrm{c}^{-k i t^{+}}$HSCs, whole bone marrow cells were incubated with phycoerythrin-conjugated anti-CD150 (TC15-12F12.2; BioLegend), FITC-conjugated anti-CD48 (HM48-1; BioLegend), FITC-conjugated antiCD41 (MWReg30; BD Pharmingen), APC-conjugated anti-Sca-1 (E13-6.7) and biotin-conjugated anti-c-kit (2B8) antibodies, in addition to FITC-conjugated antibodies against Ter119, B220 (6B2), Gr-1 (8C5) and CD2 (RM2-5). HSCs were frequently pre-enriched by selecting $\mathrm{c}-\mathrm{kit}^{+}$cells using paramagnetic microbeads and autoMACS (Miltenyi Biotec). All flow cytometry was performed on a FACSVantage SE-dual laser, three-line flow cytometer (Becton-Dickinson).

BrdU staining. Staining for BrdU on slides was performed as previously described using an anti-BrdU antibody (clone BU1/75, Accurate Chemical and Scientific Corp. ${ }^{15}$. HSCs were sorted and then resorted (to ensure purity) onto glass slides in 25-100-cell spots, allowed to dry for $1 \mathrm{~h}$ and stored at $-80^{\circ} \mathrm{C}$ for up to 4 weeks. Slides were thawed at room temperature $\left(23^{\circ} \mathrm{C}\right)$ for $15 \mathrm{~min}$, fixed in $70 \%$ ethanol at $-20{ }^{\circ} \mathrm{C}$ for $30 \mathrm{~min}$, rinsed in $0.1 \mathrm{M}$ phosphate buffer twice, incubated in $2 \mathrm{M} \mathrm{HCl}$ with $0.8 \%$ Triton in phosphate buffer for $30 \mathrm{~min}$, incubated in $0.1 \mathrm{M}$ sodium borate ( $\mathrm{pH} 8.5$ ) for $15 \mathrm{~min}$, and rinsed in $0.1 \mathrm{M}$ phosphate buffer at room temperature, then at $37^{\circ} \mathrm{C}$, and finally at room temperature again. The slides were then incubated with $0.3 \%$ Triton in $0.1 \mathrm{M}$ phosphate buffer supplemented with $5 \%$ goat serum for $1 \mathrm{~h}$ at $4{ }^{\circ} \mathrm{C}$. Slides were incubated at $4{ }^{\circ} \mathrm{C}$ overnight with a primary anti-BrdU antibody that specifically recognizes BrdU and CldU but does not recognize IdU (clone BU1/75, Accurate Chemical and Scientific Corp.). Slides were then rinsed in $0.1 \mathrm{M}$ phosphate buffer twice and were incubated with Alexa488-conjugated goat anti-rat IgG (InvitrogenMolecular Probes) for $2 \mathrm{~h}$ at room temperature. Slides were rinsed in $0.1 \mathrm{M}$ phosphate buffer twice and were incubated with DAPI for $1 \mathrm{~h}$ at room temperature. Finally, slides were rinsed 3 times in $0.1 \mathrm{M}$ phosphate buffer, the excess buffer was shaken off and the slides were mounted in $70 \%$ glycerol in PBS. Images were gathered using an Olympus BX-51 fluorescence microscope equipped with a Cooke Pixelfly CCD camera.

In some experiments, BrdU incorporation was measured by flow cytometry using an antibody directly conjugated to APC (APC BrdU flow kit, BD Pharmingen). Sorted samples were fixed and permeabilized according to manufacturer's instructions, incubated in $2 \mathrm{M} \mathrm{HCl}$ for $30 \mathrm{~min}$ at room temperature, neutralized in $0.1 \mathrm{M}$ sodium borate ( $\mathrm{pH} 8.5$ ), washed in $0.5 \%$ Triton in DPBS, stained with anti-BrdU APC and resuspended in DAPI $\left(10 \mu \mathrm{g} \mathrm{ml}^{-1}\right)$ before FACS analysis. All flow cytometry was performed on a FACSVantage SE-dual laser, three-line flow cytometer (Becton-Dickinson).

CldU and IdU double-labelling. Slides were processed as for BrdU analysis with the addition of a subsequent set of staining steps using a second anti-IdU antibody (clone B44, BD Pharmingen) that does not recognize CldU. Slides were incubated at room temperature in anti-IdU antibody for 2-3 h. Immunofluorescence was developed using a Cy3-conjugated anti-mouse IgG secondary antibody with minimal cross reactivity to rat IgG (Jackson Immunoresearch). Cells isolated from mice that had only received CldU, had only received IdU, or had received neither were processed in parallel with experimental samples and demonstrated no cross-reactivity or background staining from primary or secondary antibodies.

Analysis of cell cycle distribution and cell death in HSCs. Cell cycle distribution was analysed by Hoechst 33342 (Invitrogen-Molecular Probes) staining. $\mathrm{CD} 150^{+} \mathrm{CD} 48^{-} \mathrm{CD} 41^{-}$lineage ${ }^{-} \mathrm{Sca}-1^{+} \mathrm{c}-\mathrm{kit}^{+} \mathrm{HSC}$ were sorted and resorted into ice-cold $70 \%$ ethanol and stored at $-20{ }^{\circ} \mathrm{C}$ overnight. Cells were resuspended in PBS containing $0.02 \mathrm{mg} \mathrm{ml}^{-1}$ Hoechst 33342, incubated for $30 \mathrm{~min}$ and analysed by flow cytometry using an ultraviolet laser.

For activated caspase- 3 staining, frozen slides bearing sorted CD $150{ }^{+} \mathrm{CD} 48$ CD $41^{-}$lineage ${ }^{-} \mathrm{Sca}-1^{+} \mathrm{c}^{-\mathrm{kit}^{+}}$HSCs or sections through embryonic day 11 mouse forebrain were thawed at room temperature for $10 \mathrm{~min}$, fixed at room temperature in $10 \%$ buffered neutral formalin (VWR) for $10 \mathrm{~min}$, rinsed in $0.1 \mathrm{M}$ phosphate buffer twice and blocked with $0.3 \%$ Triton in $0.1 \mathrm{M}$ phosphate buffer supplemented with $5 \%$ goat serum for $1 \mathrm{~h}$ at $4{ }^{\circ} \mathrm{C}$. Slides were then incubated with anti-activated caspase- 3 antibody (BD Pharmingen) at room temperature for $2 \mathrm{~h}$, and rinsed in $0.1 \mathrm{M}$ phosphate buffer twice and incubated with Alexa488conjugated goat anti-rabbit IgG (Invitrogen-Molecular Probes) for $1 \mathrm{~h}$ at room temperature. The slides were then rinsed in $0.1 \mathrm{M}$ phosphate buffer twice and incubated with DAPI for $30 \mathrm{~min}$ at room temperature. Finally, slides were rinsed three times in $0.1 \mathrm{M}$ phosphate buffer, the excess buffer was shaken off and the slides were mounted in 70\% glycerol in PBS. Images were collected using a fluorescence microscope.

To test for the incorporation of BrdU due to DNA repair, BrdU was administered to adult mice for $12 \mathrm{~h}$ followed by irradiation with $100 \mathrm{rad}$ from a Gammacell40 Extractor (MDS Nordion) followed by $48 \mathrm{~h}$ of further BrdU administration. $\mathrm{CD} 150^{+} \mathrm{CD} 48^{-} \mathrm{CD} 41^{-}$lineage ${ }^{-} \mathrm{Sca}-1^{+} \mathrm{c}-\mathrm{kit}^{+}$HSCs were then sorted onto slides and stained for BrdU as described above.

Mathematical models of BrdU uptake and retention. To model the uptake and retention of BrdU in a population of stem cells we assumed that for days 0 through to $T$ the stem cells are exposed to adequate BrdU so that cells incorporate BrdU when they divide. On the basis of our data, a random $6.0 \%$ of HSCs enter the cell cycle each day. At day $T$, the BrdU supply is removed and the level of BrdU incorporated into the chromosomes decreases for every cell division after day $T$. The rate at which BrdU is diluted from cells during this chase period depends on how the cells segregate their chromosomes. If chromosomes segregate randomly then, irrespective of whether stem cells divide asymmetrically or symmetrically with respect to daughter-cell fate, BrdU-labelled chromosomes will stochastically become diluted over time: on average, the BrdU label will be diluted by half during each round of division and multiple divisions will be required to dilute the BrdU label to the point at which it is no longer detectable by immunohistochemistry. This is modelled as case 1 (see below) for asymmetrically dividing cells. In contrast, according to the immortal strand model $^{2}$, stem cells divide asymmetrically under steady-state conditions and BrdU is preferentially incorporated into newly synthesized DNA strands that are asymmetrically segregated into differentiating daughter cells with each round of division. Under these assumptions, modelled as case 2 (see below), stem cells retain only the unlabelled older DNA strands once BrdU is withdrawn.

Case 1: random segregation of chromosomes. When chromosomes are allowed to segregate randomly, BrdU could be incorporated into one or two DNA strands within each chromosome, depending on the number of times a stem cell divides during the period of BrdU incorporation and the way in which the chromosomes segregate. To model the rate of BrdU incorporation: $y_{0}$ represents the fraction of cells without BrdU; $y_{1}$ represents the fraction of cells with one strand $\mathrm{BrdU}^{+}$after only one division; $y_{2}$ represents the fraction of cells with both strands $\mathrm{BrdU}^{+}$ after two or more divisions; and $\alpha$ represents the proliferation rate of stem cells (we observed $6.0 \%$ of HSCs enter the cell cycle per day). Cell death was not incorporated into this model because we did not observe significant cell death or changes in HSC frequency during the experiments. The equations for uptake are as follows:

$$
\begin{gathered}
\frac{\mathrm{d} y_{0}}{\mathrm{~d} t}=-\alpha y_{0} \\
\frac{\mathrm{d} y_{1}}{\mathrm{~d} t}=-\alpha y_{1}+\alpha y_{0} \\
\frac{\mathrm{d} y_{2}}{\mathrm{~d} t}=\alpha y_{1}
\end{gathered}
$$

In equation (1), cells leave the $y_{0}$ population when they divide. In equation (2), cells from the $y_{0}$ population are added into the $y_{1}$ population through the incorporation of BrdU into one of the DNA strands. Cells leave the $y_{1}$ population when they divide. In equation (3), cells from the $y_{1}$ population are added into the $y_{2}$ population through further incorporation of BrdU through cell division.

To determine the frequency of $\mathrm{BrdU}^{+}$stem cells at any time after the addition of BrdU, we solve the system of ordinary differential equations, with all HSCs initially being unlabelled before BrdU administration. Similar equations have been used previously to model BrdU incorporation and depletion from other cells ${ }^{22}$.

At day $T$ (when BrdU is removed), we determine the total number of $\mathrm{BrdU}^{+}$ stem cells by adding the $y_{1}$ and $y_{2}$ populations. Cells in the $y_{1}$ population have a BrdU level of 1 , whereas $y_{2}$ cells have a BrdU level of up to 2. To model the rate at which these cells lose BrdU during subsequent divisions after removing BrdU, we used the following: $y_{10}$ represents the fraction of cells, initially with one labelled DNA strand, that undergoes zero divisions after day $T ; y_{11}$ represents the fraction of cells, initially with one labelled DNA strand, that undergoes one division after day $T ; y_{1 N}$ represents the fraction of cells, initially with one labelled DNA strand, that undergoes $N$ divisions after day $T ; y_{20}$ represents the fraction of cells, initially with two labelled DNA strands, that undergoes zero divisions after day $T ; y_{21}$ represents the fraction of cells, initially with two labelled DNA strands, that 
undergoes one division after day $T ; y_{2 N}$ represents the fraction of cells, initially with two labelled DNA strands, that undergoes $N$ divisions after day $T$. Cells move from $y_{1(k-1)}$ to $y_{1 k}$ at the proliferation rate $\alpha$ (cells move in $y_{2 k}$ similarly). The equations for dilution of the BrdU label are:

$$
\begin{gathered}
\frac{\mathrm{d} y_{10}}{\mathrm{~d} t}=-\alpha y_{10} \\
\frac{\mathrm{d} y_{11}}{\mathrm{~d} t}=-\alpha y_{11}+\alpha y_{10} \\
\frac{\mathrm{d} y_{1 N}}{\mathrm{~d} t}=\alpha y_{1(N-1)} \\
\frac{\mathrm{d} y_{20}}{\mathrm{~d} t}=-\alpha y_{20} \\
\frac{\mathrm{d} y_{21}}{\mathrm{~d} t}=-\alpha y_{21}+\alpha y_{20} \\
\frac{\mathrm{d} y_{2 N}}{\mathrm{~d} t}=\alpha y_{2(N-1)}
\end{gathered}
$$

With each cell division, the cell's BrdU level is decreased by half. For instance, cells in $y_{10}$ have a BrdU level of 1 , cells in $y_{11}$ have half as much BrdU on average, and cells in $y_{12}$ have one-quarter as much BrdU on average, and so on. Similarly, cells in $y_{20}$ have a BrdU level of 2, cells in $y_{21}$ have BrdU level 1 , cells in $y_{22}$ have a BrdU level of 0.5 , and so on. We can determine the total fraction of cells that have a BrdU level that is above a minimum detection level (which can be set at any desired level in the simulations) by adding all relevant populations at any time after day $T$.

This set of ordinary differential equations is solved for initial conditions that are determined by the observed results of the BrdU incorporation at time $T$. In this way, it is possible to plot the frequency of $\mathrm{BrdU}^{+} \mathrm{HSC}$ over time, depending on whether it takes 1, 2, 3 or 4 rounds of division to dilute BrdU to the point at which it is no longer detectable (Fig. 3a). Note that one round of division corresponds to a 2 -fold dilution of $\mathrm{BrdU}$ whereas four rounds of division correspond to 16-fold dilution. Our empirical data indicate that approximately three rounds of division are required to dilute $\mathrm{BrdU}$ to the point that it is no longer detectable in HSCs.

Case 2: asymmetric chromosome segregation. In this model, only one strand of DNA within each chromosome in stem cells can contain BrdU, irrespective of how long BrdU is administered (although the proportion of labelled stem cells will increase over time). In case 2, the equations for uptake are equation (1) (see 'case 1') and:

$$
\frac{\mathrm{d} y_{1}}{\mathrm{~d} t}=\alpha y_{0}
$$

In equation (4), cells from the $y_{0}$ population are added into the $y_{1}$ population through the incorporation of BrdU into one of the DNA strands.

As before, to determine the proportion of cells within each population at the end of day $T$, we solve the system of ordinary differential equations with initial conditions corresponding to the case in which initially all HCSs are unlabelled before BrdU administration. Cells in population $y_{0}$ do not contain any BrdU, whereas those in $y_{1}$ have a BrdU level of one.

To study the process by which cells lose BrdU label, we took all cells containing BrdU at day $T$ (all cells in population $y_{1}$ and monitored BrdU loss through cell division in the absence of BrdU). In this case, the asymmetric segregation of chromosomes means that all dividing stem cells will lose all BrdU label in a single division in the absence of BrdU. Therefore, the fraction of stem cells that remain $\mathrm{BrdU}^{+}$at time $T$ simplifies to the fraction of $\mathrm{BrdU}^{+} \mathrm{HSC}$ that do not divide after removing BrdU. Cells move from $y_{10}$ (and lose their BrdU label) at the proliferation rate $\alpha$. The equation for loss of BrdU label is:

$$
\frac{\mathrm{d} y_{10}}{\mathrm{~d} t}=-\alpha y_{10}
$$

This differential equation with initial condition $y_{10}(0)=y_{1}(T)$ determines the fraction of HSCs that retain BrdU over time, according to the immortal strand hypothesis.

22. Bonhoeffer, S., Mohri, H., Ho, D. \& Perelson, A. S. Quantification of cell turnover kinetics using 5-bromo-2'-deoxyuridine. J Immunol. 164, 5049-5054 (2000). 